\title{
Management of Free-Floating Thrombus in the Vertebral Artery in a Middle-Aged Smoker with Secondary Polycythemia Vera
}

\author{
Ali Alhashim' \\ Kawther Hadhiah (D) \\ Sarah A Itani ${ }^{2}$ \\ Mohammed Alshurem ' \\ Majed Alabdali \\ Danah Aljaafari ${ }^{\prime}$ \\ Mustafa AIQarni \\ 'Neurology Department, College of \\ Medicine, Imam Abdulrahman Bin Faisal \\ University, Dammam, 34212, Saudi \\ Arabia; ${ }^{2}$ College of Medicine, Imam \\ Abdulrahman Bin Faisal University, \\ Dammam, 342/2, Saudi Arabia
}

\begin{abstract}
Free-floating thrombus (FFT) of the cervicocranial arteries is a rare neurovascular condition. Up to now, there is no standardized definition for FFT. Therefore, FFT is occasionally mistaken for intraluminal thrombus (ILT) or smooth mural thrombus. The most precise and ideal definition of FFT would be a long-extended intraarterial thrombus that is attached to the arterial wall with its one end, while its other end is surrounded by blood flow and moves freely with the cardiac cycle. FFT usually manifests as an ischemic stroke, thus it is considered as an emergency case. Herein, we report a rare case of symptomatic FFT in the left vertebral artery extending from V0 to V2 segments in a middle-aged smoker, who presented with multiple embolic strokes in different territories of posterior circulation and was successfully treated medically. This case sheds light on the challenges of the clinical approach of FFT in the vertebral artery and it is an attempt to draw attention to the necessity of conducting a large-scale study to find out the ideal approach to manage such conditions.
\end{abstract}

Keywords: stroke, free-floating thrombus, FFT, secondary polycythemia vera, PV, dual antithrombotic

\section{Introduction}

Free-floating thrombosis (FFT) is an uncommon etiology of ischemic stroke and its backbone literature is based on case reports and series, where most papers primarily discuss internal carotid artery free-floating thrombus. In the recent literature there have been extremely few reported cases about vertebral artery FFT. Nevertheless, these are regrettably inconsistent due to the lack of a unified morphological definition and standardized radiological diagnostic features of the FFT. FFT has a male predilection, with a 2:1 male-to-female ratio, and it is more common in younger age groups. ${ }^{3}$ The manifestation in middle age and the consequences of neurological deficits mean FFT is a matter of great significance to be studied. The exact pathogenesis of FFT is still uncertain. Hypercoagulability and atherosclerosis are the highest probable etiologies in most reports. ${ }^{2,3}$ The mechanism behind atherosclerosis is debated to be either due to plaque erosion or plaque rupture. Other causes such as dissection and drug abuse are rarely reported. ${ }^{7,9}$ Whereas FFT could be asymptomatic and found incidentally, ${ }^{1,3}$ patients typically present with acute neurological deficits as a sequela of cerebral ischemic insult in context of transient ischemic attack (TIA) or well-established stroke. Some patients even develop evolving or current stroke. ${ }^{3,5}$ In this paper we describe a case of symptomatic FFT in the vertebral artery and we highlight the diagnostic as well as the therapeutic challenges that might be faced in approaching FFT.
Correspondence: Ali Alhashim Neurology Department, College of Medicine, Imam Abdulrahman Bin Faisa University, Dammam, 34212 , Saudi Arabia Tel +966509444833

Email ali_al_hashem@hotmail.com; ahalhashem@iau.edu.sa 


\section{Case Presentation}

A 47-year-old Indian man who works as a private driver presented to our emergency department with acute vertigo, nausea, and vomiting after he had eaten in a public restaurant. He had been initially misdiagnosed as a case of gastroenteritis and discharged home. On the following day, he presented again with acute-onset focal neurologic deficits, described as a feeling of imbalance and falling to the right side, associated with a difficulty in speaking. The patient has not reported any history of headache nor other focal neurological deficits. He was a smoker (20 pack-years), however substance abuse, including drug and alcohol, was credibly denied. He was not known to have any other medical illnesses nor did he take any medications. Social as well as family history were unremarkable. Upon examination, the patient was conscious and oriented to time, place, and person. He exhibited right limb ataxia as seen on finger-nose and heel-shin tests as well as dysarthria, with a total NIH stroke scale (NIHSS) of 3. His blood pressure was slightly elevated (155/94), respiratory rate was 23 /minute, temperature was $36.5 \mathrm{C}^{\circ}$, and pulse was $80 \mathrm{bpm}$. Laboratory tests were done at presentation (Table 1), which revealed an elevated hemoglobin $(18.7 \mathrm{~g} / \mathrm{dL})$, hematocrit $(62.5 \%)$, partial thromboplastin time (43.2 seconds), and Prothrombin time (14.4 seconds). Due to a high suspicion of acute cerebrovascular insult, a non-contrast cerebral neuroimaging via computed tomography (CT) (Figure 1) was performed and showed multiple areas of acute and subacute ischemic stroke in different territories including the posterior cerebral artery (PCA), superior cerebellar artery (SCA), posterior inferior cerebellar artery (PICA), and anterior inferior cerebellar artery (AICA) bilaterally. The cerebral CT-angiogram (CTA), from arch to vertex, showed a long segment of filling defect in the left vertebral artery, extending from V0 up to V2 segments, which is suggestive of a long, fresh, free-floating thrombus with the classical donut sign appearance (Figures 2 and 3). Based on clinico-radiographic findings, a diagnosis of multiple cerebral as well as cerebellar ischemic strokes due to arterio-arterial embolization was made. Acute management of stroke with thrombolysis and endovascular therapy was ineligible since the presentation was outside the thrombolysis window (over 24 hours from symptoms onset). To characterize the etiology of stroke we conducted a transthoracic echocardiogram (TTE) and long-term electrocardiogram (ECG) monitoring, which could not detect
Table I Blood Work-Up Done for the Patient at Presentation

\begin{tabular}{|c|c|c|}
\hline Test & Result & $\begin{array}{l}\text { Reference } \\
\text { Range }\end{array}$ \\
\hline \multicolumn{3}{|l|}{ Hematology } \\
\hline $\mathrm{RBC}(\mathrm{Mil} / \mu \mathrm{L})$ & 6.03 & $4.7-6.1$ \\
\hline 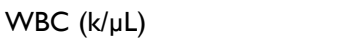 & 9.3 & $4.1-11.0$ \\
\hline Segs (\%) & 57.1 & $40-75$ \\
\hline Lymphocyte (\%) & 30.5 & $20-45$ \\
\hline Hemoglobin (g/dL) & 18.7 & $13.0-18.0$ \\
\hline Hematocrit (\%) & 62.5 & $42-52$ \\
\hline Platelets $(\mathrm{k} / \mu \mathrm{L})$ & 225 & $140-450$ \\
\hline \multicolumn{3}{|l|}{ Blood chemistry } \\
\hline Random blood sugar (mg/dL) & 84 & $70-140$ \\
\hline Hemoglobin AIC (\%) & 6.4 & $<6 \%$ \\
\hline Total cholesterol (mg/dL) & 200 & $<200$ \\
\hline LDL (mg/dL) & 170 & $<100$ \\
\hline \multicolumn{3}{|l|}{ Coagulation } \\
\hline PT (s) & 14.4 & $10.0-14.0$ \\
\hline aPTT (s) & 43.2 & $20.0-40.0$ \\
\hline INR & 1.01 & \\
\hline \multicolumn{3}{|l|}{ Inflammatory markers } \\
\hline ESR $(\mathrm{mm} / \mathrm{h})$ & 5 & $0-20$ \\
\hline Quantitative CRP (mg/dL) & 0.2 & $0.1-0.5$ \\
\hline \multicolumn{3}{|l|}{ Autoimmune work-up } \\
\hline Antinuclear antibodies (ANA) & $\mathrm{I}: 80$ & $<1: 80$ \\
\hline Anti-double stranded DNA & $<1: 10$ & $<1: 10$ \\
\hline $\begin{array}{l}\text { Lupus anticoagulant screen } \\
\text { ratio }\end{array}$ & 0.8 & $<1.2$ \\
\hline Anti-cardiolipin IgM & 1.3 & $<20$ \\
\hline Anti-cardiolipin IgG & 4.5 & $<20$ \\
\hline
\end{tabular}

Abbreviations: RBC, Red blood cell; WBC, white blood cell; aPTT, Activated partial thromboplastin time; PT, Prothrombin time; INR, International normalized ratio; s, seconds; DNA, Deoxyribonucleic acid; IgG, Immunoglobulin G; IgM, Immunoglobulin M; ESR, Erythrocyte sedimentation rate; CRP, C-reactive protein.

any evidence of cardiogenic embolic sources. Particularly, there was no evidence of atrial fibrillation, intracardial thrombus, regional wall motion abnormalities, atrial septal anomalies, or valvular dysfunction. Furthermore, extensive laboratory investigations, including thrombophilia and vasculitis screening, were unremarkable. Hematological colleagues were consulted and they attributed blood changes to heavy smoking and made a diagnosis of secondary polycythemia vera due to smoking.

The patient was admitted to the stroke unit for close monitoring and was started on dual antiplatelet with aspirin $100 \mathrm{mg}$ and clopidogrel $75 \mathrm{mg}$ as well as 


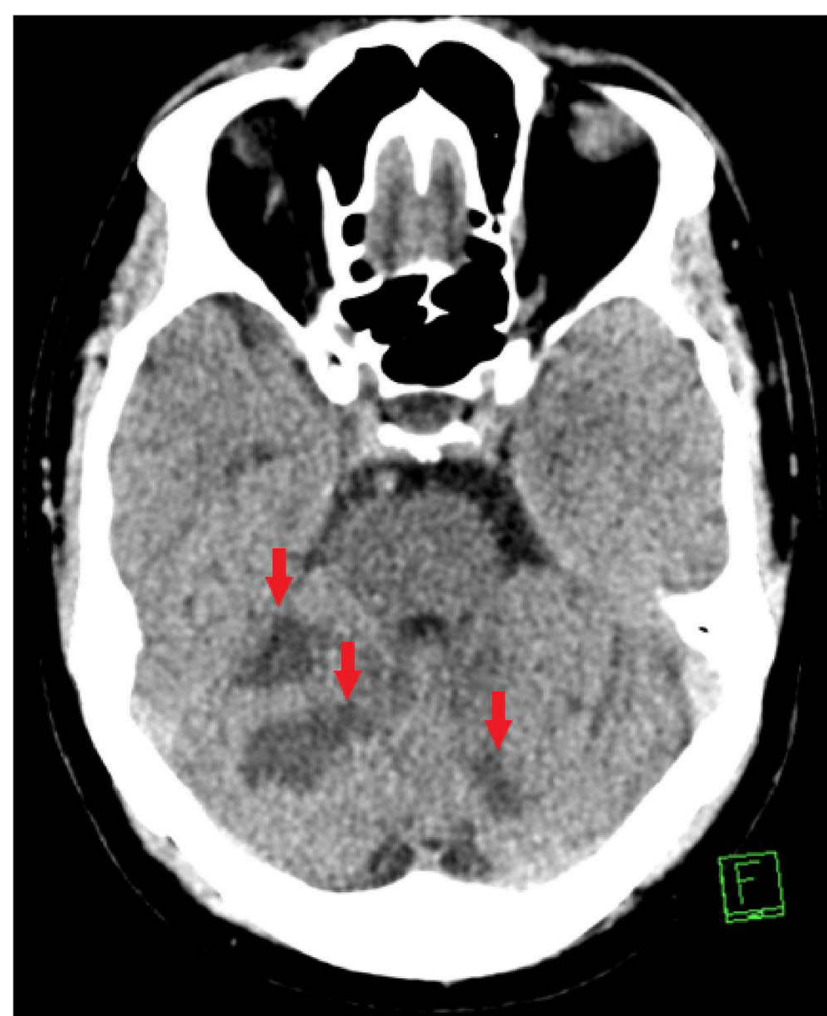

Figure I An axial view of a non-contrast computed tomography scan showing multiple areas of acute and subacute ischemic strokes in different territories.

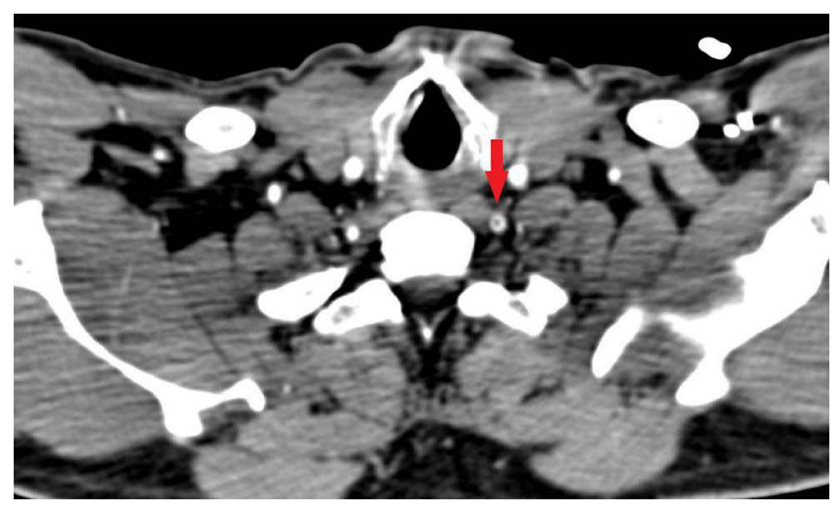

Figure 2 An axial view of computed tomography angiography scan showing a donut sign in the left vertebral artery, suggestive of free-floating thrombus at the level of $\mathrm{VI}$.

thrombosis prophylaxis anticoagulation with enoxaparin $40 \mathrm{mg}$ and atorvastatin $80 \mathrm{mg}$. He was also maintained on good hydration (100-120 mg/hour) and underwent phlebotomy. In the follow-up laboratory test, the hemoglobin and hematocrit fell to $14.2 \mathrm{~g} / \mathrm{dL}$ and $42.8 \%$, respectively. Throughout his stay in the hospital, he remained stable with evident improvement in his symptoms (NIHSS dropped to 1 point). The CTA follow-up (Figure 4) and doppler sonography showed complete canalization of the thrombus in the left vertebral artery. In addition, the

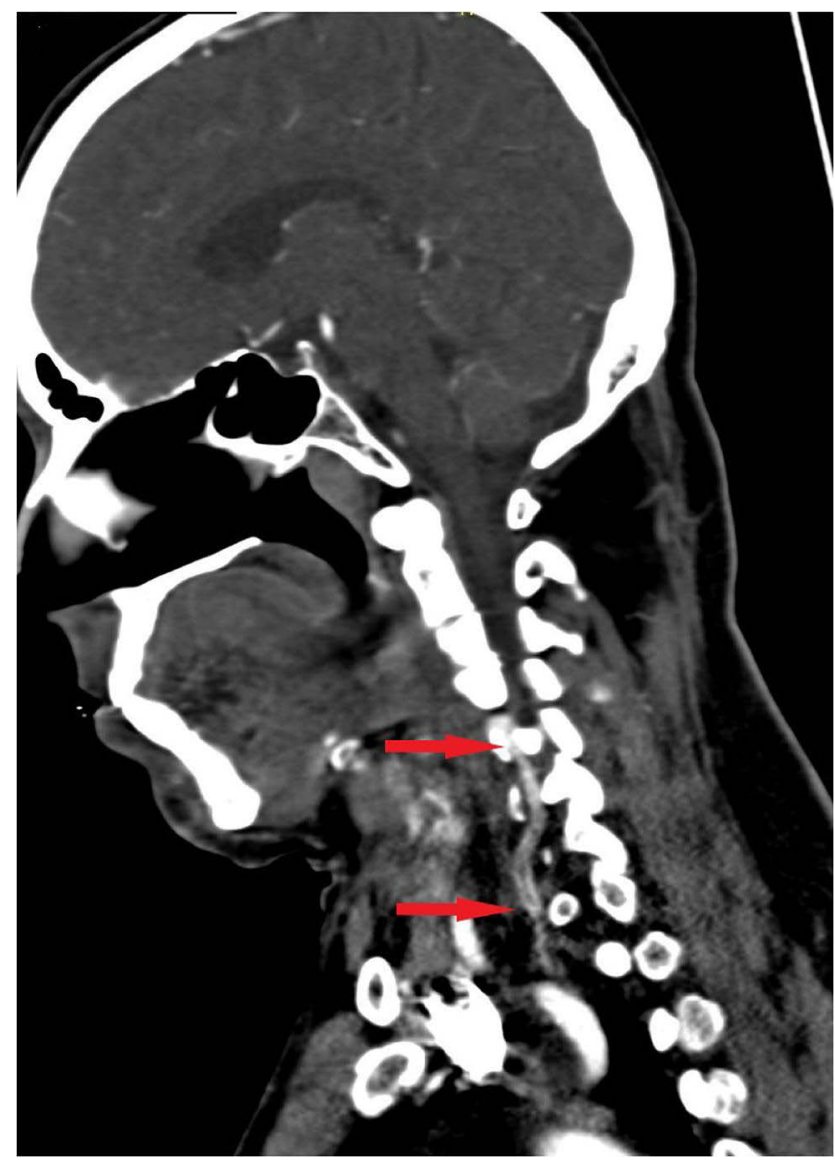

Figure $3 \mathrm{~A}$ coronal view of computed tomography angiography scan showing a long, fresh, free-floating thrombus in VI and V2.

follow-up neuroimaging via magnetic resonance imaging (MRI) showed no new ischemic insult in comparison to the initial cerebral CT (Figure 5).

\section{Discussion}

FFT of cervicocranial arteries is an uncommon entity. Its incidence has been reported to be $1.53 \%$ among stroke patients. $^{5}$ The occurrence of FFT was reported in the vast majority of case reports to be in the anterior extracranial circulation and more commonly in the internal carotid artery (ICA) $(75 \%){ }^{3}$ In contrast, extremely few vertebral artery FFT (VA-FFT) cases were reported.

FFT is an acute neurological emergency that requires immediate attention and is usually symptomatic., ${ }^{3,4}$ However, few asymptomatic cases were reported in the literature. ${ }^{1}$ Our patient was symptomatic with recurrent strokes in the posterior circulation prior to therapy initiation.

Atherosclerosis and hypercoagulability are considered the likely etiologies in most reports. ${ }^{2,3}$ In this case, the underling etiopathogenesis of FFT was the hyperviscosity due to 


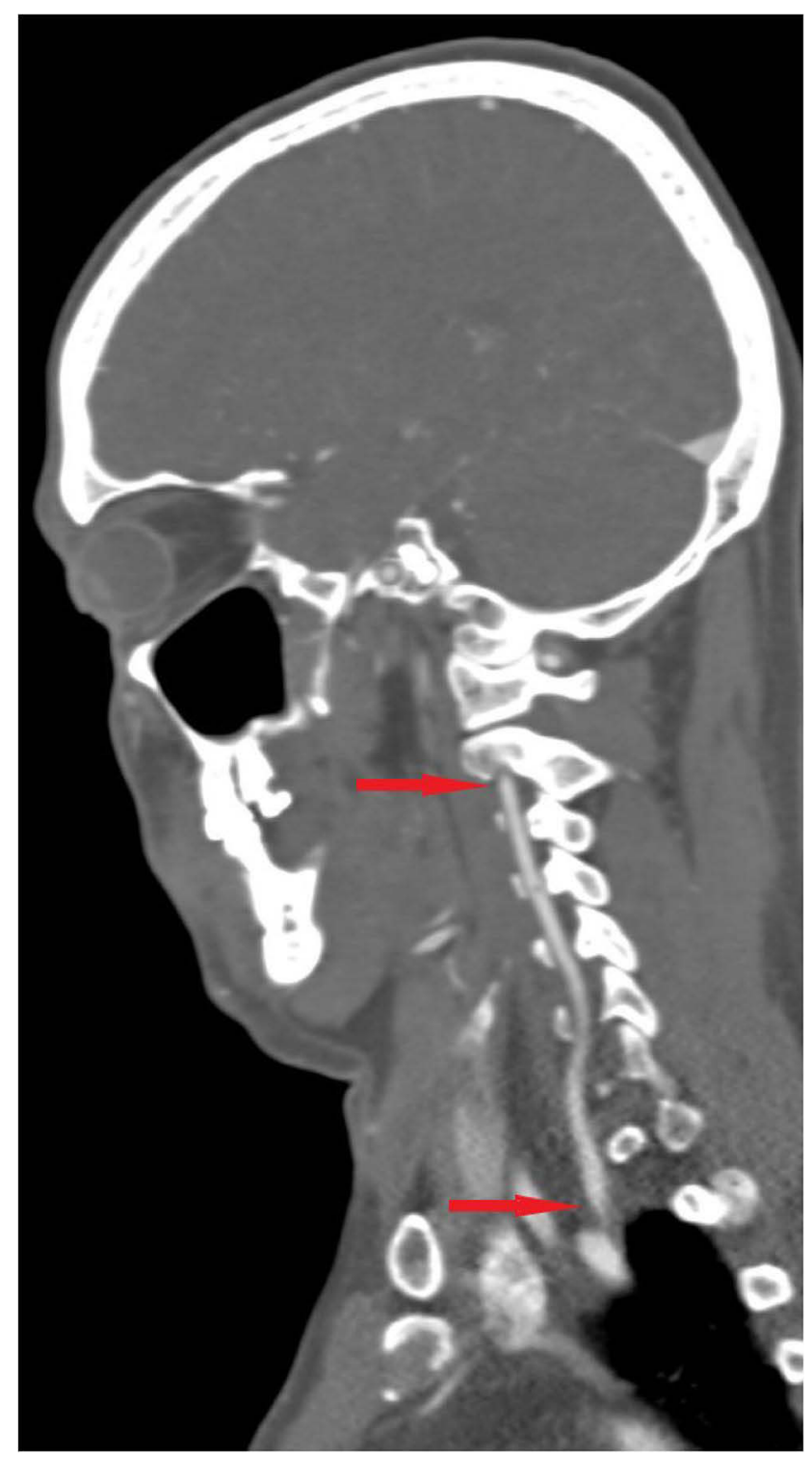

Figure $4 \mathrm{~A}$ coronal view of computed tomography angiography scan, showing complete canalization of the thrombus in the left vertebral artery 7 days post initiation of therapy.

increased cellular component of blood in the context of secondary polycythemia vera $(\mathrm{PV})$, which results in a hypercoagulable status. ${ }^{6}$ To our knowledge, there have not been any reports about the association of FFT and PV. So, the rarity of FFT occurrence in vertebral artery combined with the association with PV gives this case some uniqueness.

CTA is helpful in diagnosing FFT as it shows the characteristic donut $\operatorname{sign}^{8}$ and the length of the thrombus. Moreover, it can aid in estimating the associated stenosis in these patients; a measurement threshold of more than $3.8 \mathrm{~mm}$ on CTA was found to be highly specific and sensitive for FFT diagnosis. ${ }^{12}$ Further delineation of the free-floating thrombus can be done by doppler ultrasound

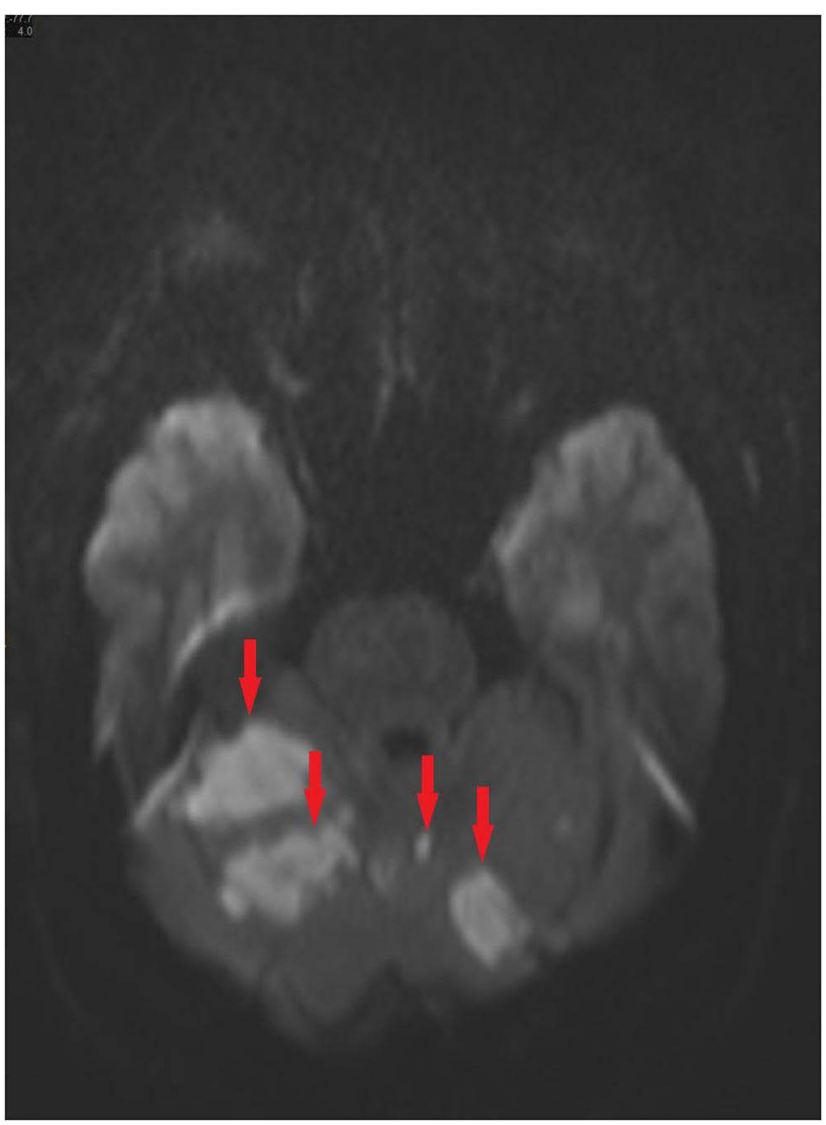

Figure 5 An axial view of a follow-up magnetic resonance imaging scan showing multiple areas of acute and subacute ischemic strokes in different cerebellar territories, with no additional cerebrovascular insults to the initial non-contrast computed tomography scan.

of the carotid or vertebral arteries, as it is handy and easily available. Duplex scan and digital subtraction angiography (DSA) had sensitivities of $62.5 \%$ and $100 \%$, respectively. ${ }^{4}$

In the published literature there has been a diversity in the therapeutic approach with satisfactory outcomes reported. These approaches vary from medical therapy with either single or dual antithrombotic (anticoagulation and/or antiplatelet) to urgent surgical intervention via carotid endarterectomy (CEA) or endovascular therapy (EVT). Up to date, the best available evidence for FFT treatment would be from a systematic literature review by Fridman et al. ${ }^{5}$ It has shown a trend favoring the medical approach with anticoagulants over the surgical approach; however, due to the lack of high-quality evidence from the literature published hitherto, no robust conclusion could be made. Likewise, a small retrospective study of FFT treatment in the proximal aorta and supra-aortic branches tended to the medical approach. It showed $90 \%$ resolution of FFT without adverse events under medical therapy with anticoagulant and/or 
antiplatelet. ${ }^{9}$ Nevertheless, a successful surgical intervention via EVT in ICA-FFT and VA-FFT has also been reported. ${ }^{1,11}$ A published review article by Bahtti et $\mathrm{al}^{3}$ failed to show any superiority of either approach. As the therapeutic guideline for FFT has not been established yet, each patient should be approached, for the time being, individually in an interdisciplinary manner (neurology, neurosurgery, and neuroradiology) as we have done with our patient. All therapeutic options were discussed and the decision went against EVT in fear of dislodging of the thrombus, since it was very extensive. However, EVT would be the first-line therapy if spontaneous migration of the thrombus in the basilar artery occurred. Despite the favoring of anticoagulation therapy in most reported studies, we were reluctant to begin anticoagulation in fear of developing hemorrhagic transformation, as the patient had multiple moderately sized infarctions in the cerebellum. After weighing the risks against the benefits, we decided to go for dual antiplatelet therapy with aspirin and clopidogrel along with the adjuvant therapy of repetitive phlebotomy with a target hematocrit below $45 \%{ }^{10}$ and adequate hydration to reduce blood hyperviscosity and prevent progression of the thrombus. Within a few days of admission, the patient's symptoms improved dramatically and no new further events occurred. The follow-up CTA on day 7 showed resolution of FFT.

\section{Conclusion}

The significance of FFT cases involving cervicocranial arteries lies in the seriousness of the condition as well as natural age of manifestation, since it may cause a stroke in middle-aged, well-functioning patients and result in devastating disability. For that reason, the neurologist should be aware of such a special entity and its consequences, monitor the patients closely, and treat them more aggressively using either the medical or surgical approach. For further studies, we recommend more research in the form of randomized controlled clinical trials for evaluating the efficacy, safety, and duration of aggressive medical therapy. Furthermore, a head-to-head study is needed to compare the superiority of either aggressive medical therapy or invasive surgical therapy; or otherwise, the indications of each.

\section{Statement of Ethics}

The subject has provided his informed consent for the creation of this manuscript with full knowledge that his clinical findings and treatment are utilized herein for educational and peer-reviewed publication purposes. Ethical approval from the institutional review board at Imam Abdulrahman Bin Faisal University was not required to publish the details of this case.

\section{Consent}

Written informed consent was obtained from the patient for publication of this case report and any accompanying images.

\section{Disclosure}

The authors have declared no conflicts of interest for this work.

\section{References}

1. Al-Jehani H, Alhamid MA, Alkhalaf Y, Alabbas F. A case of coincidental free floating thrombus in the vertebral artery in a patient presenting with an anterior circulation stroke and literature review. Neurointervention. 2020;15(3):144-153. doi:10.5469/neuroint.20 20.00087

2. Monteiro A, Cunha Y, Cortez CM, Sauvageau E, Hanel R. Spontaneous resolution of carotid stenosis with free floating thrombus: a brief overview of possible mechanisms and management. Cureus. 2020;12(4):e7602.

3. Bhatti AF, Leon LR, Labropoulos N, et al. Free-floating thrombus of the carotid artery: literature review and case reports. J Vasc Surg. 2007;45(1):199-205. doi:10.1016/j.jvs.2006.09.057

4. Ferrero E, Ferri M, Viazzo A, et al. Free-floating thrombus in the internal carotid artery: diagnosis and treatment of 16 cases in a single center. Ann Vasc Surg. 2011;25(6):805-812. doi:10.1016/j. avsg.2011.02.030

5. Fridman S, Lownie SP, Mandzia J. Diagnosis and management of carotid free-floating thrombus: a systematic literature review. Int J Stroke. 2019;14(3):247-256. doi:10.1177/1747493019828554

6. Artoni A, Bucciarelli P, Martinelli I. Cerebral thrombosis and myeloproliferative neoplasms. Curr Neurol Neurosci Rep. 2014;14 (11):496. doi:10.1007/s11910-014-0496-y

7. Cosenza M, Panza L, Califano AP, et al. Carotid thrombosis in a crack cocaine smoker woman. Case Rep Vasc Med. 2020;2020:4894825.

8. Menon BK, Singh J, Al-Khataami A, Demchuk AM, Goyal M; Calgary CTA Study Group. The donut sign on CT angiography: an indicator of reversible intraluminal carotid thrombus? Neuroradiology. 2010;52(11):1055-1056. doi:10.1007/s00234-0100738-x

9. Gülcü A, Gezer NS, Men S, Öz D, Yaka E, Öztürk V. Management of free-floating thrombus within the arcus aorta and supra-aortic arteries. Clin Neurol Neurosurg. 2014;125:198-206. doi:10.1016/j. clineuro.2014.08.008

10. Berk PD, Goldberg JD, Donovan PB, Fruchtman SM, Berlin NI, Wasserman LR. Therapeutic recommendations in polycythemia vera based on Polycythemia Vera Study Group protocols. Semin Hematol. 1986;23(2):132-143.

11. Park JW, Lee DH, Choi CG, Kim SJ, Suh DC. Various endovascular approaches to the management of free floating carotid thrombi: a technical report. $J$ Neurointerv Surg. 2012;4(5):336-338. doi:10.1136/neurintsurg-2011-010023

12. Jaberi A, Lum C, Stefanski P, et al. Computed tomography angiography intraluminal filling defect is predictive of internal carotid artery free-floating thrombus. Neuroradiology. 2014;56(1):15-23.0. doi:10.1007/s00234-013-1298-7 


\section{Publish your work in this journal}

The International Medical Case Reports Journal is an international, peer-reviewed open-access journal publishing original case reports from all medical specialties. Previously unpublished medical posters are also accepted relating to any area of clinical or preclinical science. Submissions should not normally exceed 2,000 words or 4 published pages including figures, diagrams and references. The manuscript management system is completely online and includes a very quick and fair peer-review system, which is all easy to use. Visit http://www.dovepress.com/testimonials.php to read real quotes from published authors. 